\title{
Exploring the Association between Monoclonal Antibodies and Depression and Suicidal Ideation and Behavior: A VigiBase Study
}

\author{
Lotte A. Minnema ${ }^{1,2}$. Thijs J. Giezen ${ }^{2,3}$. Patrick C. Souverein ${ }^{1} \cdot$ Toine C. G. Egberts $^{1,4} \cdot$ Hubert G. M. Leufkens ${ }^{1}$. \\ Helga Gardarsdottir ${ }^{1,4,5}$
}

Published online: 8 January 2019

(c) The Author(s) 2019

\begin{abstract}
Introduction Several monoclonal antibodies (mAbs) have been linked to neuropsychiatric adverse effects in patients, including depression and suicidal ideation and behavior.

Objective The aim of this study was to quantify and characterize spontaneously reported adverse drug reactions (ADRs) of depression and suicidal ideation and behavior related to $\mathrm{mAb}$ users, and to explore a possible association with their mechanism of action.

Methods We included mAb ADRs that were reported in VigiBase, and identified those related to depression and suicidal ideation and behavior. Reporting odds ratios (RORs) were estimated for each mAb (bevacizumab as the reference) and according to their influence on the immune system (not directly targeting [reference], stimulating, or suppressing). Those suppressing the immune system were further divided into their intended indication (auto-immune diseases, cancer).

Results Overall, 2,924,319 ADRs for 44 mAbs were included; 9455 ADRs were related to depression and 1770 were related to suicidal ideation and behavior. The association was strongest for natalizumab and belimumab, both for depression (ROR 5.7, 95\% confidence interval [CI] 5.0-6.4; and ROR 5.1, 95\% CI 4.2-6.2) and suicidal ideation and behavior (ROR 12.0, 95\% CI 7.9-18.3; and ROR 20.2, 95\% CI 12.4-33.0). Those suppressing the immune system showed higher ROR, i.e. 1.9 (95\% CI 1.8-2.0) for depression and 3.6 (95\% CI 3.0-4.4) for suicidal ideation and behavior. This finding was only seen for $\mathrm{mAbs}$ used for treating autoimmune diseases.

Conclusion Depression and suicidal ideation and behavior are seen in patients using mAbs, particularly mAbs used for treating autoimmune diseases that suppress the immune system. For interpretation of these data, the indications for use and other characteristics require further consideration.
\end{abstract}

Electronic supplementary material The online version of this article (https://doi.org/10.1007/s40264-018-00789-9) contains supplementary material, which is available to authorized users.

\section{Helga Gardarsdottir}

h.gardarsdottir@uu.nl

1 Division of Pharmacoepidemiology and Clinical Pharmacology, Utrecht Institute for Pharmaceutical Sciences (UIPS), Utrecht University, PO Box 80082, 3508 TB Utrecht, The Netherlands

2 Medicines Evaluation Board, Utrecht, The Netherlands

3 Foundation Pharmacy for Hospitals in Haarlem, Haarlem, The Netherlands

4 Department of Clinical Pharmacy, University Medical Center Utrecht, Utrecht, The Netherlands

5 Department of Pharmaceutical Sciences, School of Health Sciences, University of Iceland, Reykjavík, Iceland

\section{Key Points}

Depression and suicidal ideation and behavior are reported for monoclonal antibodies (mAbs), especially for mAbs used for the treatment of patients with autoimmune diseases that suppress the immune system.

The present study provides important knowledge for future research on the relation between the different inflammatory factors and the occurrence of depression and suicidal ideation and behavior. Future studies should focus on, amongst others, indications for use, and population characteristics to characterize and quantify this potential risk. 


\section{Introduction}

In May 2015, the phase III clinical trials investigating the efficacy and safety of brodalumab, a monoclonal antibody (mAb) against the interleukin (IL)-17 receptor, in patients with psoriasis were terminated early by the pharmaceutical company [1]. The trigger for this decision was six reports of completed suicide as adverse events in the approximately 5000 patients treated with brodalumab in the clinical trial program [2], although the evaluation thereof by both the pharmaceutical company and regulatory authorities concluded that a causal relation between the use of brodalumab and suicidal ideation and behavior was unlikely [1, 2]. In May 2017, brodalumab was authorized in the European Union based on the assessment of the regulatory authorities that the efficacy of brodalumab outweighs the risks, including the potential risk of suicidal ideation and behavior [2]. Despite this conclusion, a warning was included in the product information to carefully weigh the risks and benefits of treatment with brodalumab for patients with a history of depression and/or suicidal ideations, and for patients who develop these symptoms during treatment. Furthermore, a postauthorization safety study was required with a focus on serious events of, among others, suicidal ideation and behavior. To date, a mechanism through which brodalumab may cause such events is not known. Research in animal studies has shown that IL-17, which is blocked by brodalumab, can influence neurological function and therefore modulate behavior [3-5]; however, this has not been studied in humans.

Depression and suicidal ideation and behavior have also been reported in clinical trials of other mAbs, such as belimumab, which is used to treat patients with systemic lupus erythematosus and targets against B-cell activating factor [6]. In addition, the mAbs infliximab, adalimumab, and natalizumab have, in case reports, been associated with suicidal ideation and behavior [7-11].

The aforementioned mAbs exert their intended effects through targeting different (anti-)inflammatory factors. During the past decades, the link between psychiatric disorders and autoimmune disorders has been extensively discussed [12-14]. This link is considered to be partially explained by the influence of inflammatory factors on the brain. Different meta-analyses have evaluated the contribution of inflammatory factors in the pathophysiology of major depression and suicidal ideation and behavior [15-20]. These indicate that alterations in both pro-inflammatory and anti-inflammatory factors are linked to psychiatric disorders; however, mAbs that have a mechanism of action not directly targeting the immune system have also been linked to psychiatric disorders. For example, approximately $1-10 \%$ of patients treated with trastuzumab, a human epidermal growth factor receptor 2 inhibitor used for the treatment of cancer, develop depression [21]. It should be noted that the estimated prevalence of major depression among patients with both cancer and autoimmune diseases exceed the estimated prevalence in the general population $[22,23]$. It is therefore challenging to differentiate between the underlying disease and the effect of treatment.

To date, no studies have, to our knowledge, evaluated the potential risk of depression and suicidal ideation and behavior for the group of mAbs as a whole. Therefore, the first aim of this study was to quantify and characterize spontaneously reported adverse drug reactions (ADRs) related to depression and suicidal ideation and behavior for $\mathrm{mAbs}$. In addition, the association between the mechanism of action of the mAb and spontaneously reported ADRs of depression and suicidal ideation and behavior will be explored.

\section{Methods}

\subsection{Setting and Data Source}

VigiBase, the World Health Organization global individual case safety report (ICSR) database that is maintained by the Uppsala Monitoring Centre, was used as the data source for this study [24]. As of December 2017, over 16 million case reports of ADRs have been submitted since the start of data collection in 1968. The ICSRs are first reported by healthcare professionals and patients to more than 120 national pharmacovigilance centers, and then transferred to VigiBase. ICSRs contain information regarding patient characteristics, suspected drugs, ADRs, and additional information relevant to the report, such as the reporter type, reporting year, and reporting region [24]. Completeness of the ICSRs is variable. ADRs are classified according to the Medical Dictionary for Regulatory Activities (MedDRA ${ }^{\circledR}$ ), and suspected drugs are classified according to the Anatomical Therapeutic Chemical (ATC) classification.

All reports included in VigiBase until December 2017 in which an $\mathrm{mAb}$ was the suspected drug were identified. Only mAbs that had been authorized by the European Medicines Agency (EMA) and/or the US FDA for 3 or more years as of December 2017 were included, accounting for a representative reflection of the ADRs that are reported. Information on the regulatory status was retrieved from the publicly available information on the FDA (http://www.fda.gov) and EMA (http://www.ema.europa.eu) websites. 


\subsection{Outcome}

Spontaneously reported ADRs related to depression and suicidal ideation and behavior were identified using the Standardized MedDRA ${ }^{\circledR}$ Query (SMQ) 'Depression and suicide/ self-injury (narrow)' [MedDRA ${ }^{\circledR}$ version 20.1]. SMQs are validated and maintained by the Maintenance and Support Services Organization and updated with each version of MedDRA ${ }^{\circledR}$ [25]. The SMQ 'Depression and suicide/selfinjury (narrow)' contains 36 preferred terms. A distinction is made in preferred terms related to depression $(n=24)$ and suicide/self-injury $(n=12)$.

\subsection{Exposure}

The association between the mAb and spontaneously reported ADRs of depression and suicidal ideation and behavior was defined in two ways. First, exposure was defined for the mAbs individually using bevacizumab as the reference. Second, mAbs were grouped by their influence on the immune system (not directly targeting the immune system, suppressing or stimulating the immune system) based on the information provided in the product information (see electronic supplementary material 1). mAbs suppressing the immune system were further stratified according to their intended indication based on their ATC code (autoimmune diseases or cancer). Information on the influence of mAbs on the immune system and their intended indication was retrieved from the product information publicly available on the FDA and/or EMA websites.

\subsection{Data Analysis}

Descriptive statistics were used to quantify the reported ADRs at the level of the drug-ADR pair. ADRs were stratified by sex (male, female), age ( $<18$ years, $18-44$ years, $45-64$ years, and $\geq 65$ years), reporting year (from the first reporting year divided into periods of 5 years), reporting region (Africa, the Americas, Southeast Asia, Europe, Eastern Mediterranean, and Western Pacific), and reporter type (healthcare professional, consumer, other). The proportions of ADRs for depression and suicidal ideation and behavior were calculated by dividing the number of depression or suicidal ideation and behavior ADRs by the total number of reported ADRs within the stratum.

The strength of the association between mAbs and neuropsychiatric effects was expressed as the reporting odds ratio (ROR) with 95\% confidence intervals (CIs) [26, 27]. RORs were estimated for the ADRs related to depression and suicidal ideation and behavior separately. For analysis of the individual $\mathrm{mAbs}$, bevacizumab was used as the reference as it does not directly target the immune system and has been widely used for years, and was therefore considered to have an established safety profile. For the analysis of the mAbs grouped by their influence on the immune system, mAbs not directly targeting the immune system were used as the reference. In addition, for the subgroup analysis stratified by the intended indication, mAbs not directly targeting the immune system were used as the reference.

All data were analyzed using SPSS for Windows, version 24.0 (IBM Corporation, Armonk, NY, USA).

\section{Results}

In VigiBase, 3,048,884 ADRs were identified for 139 different mAbs as suspected drugs; 124,565 ADRs for 95 $\mathrm{mAbs}$ were excluded because they were reported for mAbs that had not been authorized by the FDA and/or EMA for 3 or more years as of December 2017. The study population therefore comprised $44 \mathrm{mAbs}$ (active substances), for which a total of $1,048,576$ ICSRs were filed, representing 2,924,319 drug-ADR pairs, of which $9455(0.32 \%)$ were related to depression and $1770(0.06 \%)$ were related to suicidal ideation and behavior.

The proportion of suicidal ideation and behavior ADRs was comparable between men and women $(0.07 \%$ and $0.06 \%$, respectively), whereas the proportion of depression ADRs was higher in women compared with men $(0.35 \%$ and $0.27 \%$, respectively) (Table 1 ). The highest proportion of depression and suicidal ideation and behavior ADRs was observed in the age range between 18 and 64 years, at $0.35 \%$ and $0.08 \%$, respectively (Table 1 ). The highest proportion of depression ADRs originated from the Americas region $(0.36 \%)$, followed by the European region $(0.17 \%)$. No regional differences were seen in the proportion of suicidal ideation and behavior ADRs. The proportion of ADRs involving depression increased over time, from $0.11 \%$ in the period between 2000 and 2004, to $0.28 \%$ in the period between 2015 and 2017, whereas the frequency of suicidal ideation and behavior remained at approximately $0.06 \%$. Among consumer reports, the proportion of depression ADRs was higher $(0.44 \%)$ than among reports of healthcare professionals $(0.23 \%)$, whereas for suicidal ideation and behavior, the proportion was comparable $(0.05 \%$ and $0.07 \%$ ).

For seven mAbs, no ADRs were reported for depression, and for $17 \mathrm{mAbs}$, no suicidal ideation and behavior ADRs were reported; therefore, the ROR could not be estimated for these mAbs.

For depression, the association (relative to bevacizumab) was strongest for natalizumab (ROR 5.7, 95\% CI 5.0-6.4), followed by belimumab (ROR 5.1, 95\% CI 4.2-6.2) (Fig. 1). Furthermore, RORs were calculated for different groups of mAbs based on their influence on the immune system (Fig. 2). For depression, the association 
Table 1 Characteristics of depression, suicidal ideation and behavior, and all adverse drug reactions reported for the monoclonal antibodies

\begin{tabular}{|c|c|c|}
\hline & Depression ADRs (\%) & $\begin{array}{l}\text { Suicidal ideation and } \\
\text { behavior ADRs (\%) }\end{array}$ \\
\hline Total $(n=2,924,319)$ & $9455(0.32)$ & $1770(0.06)$ \\
\hline \multicolumn{3}{|l|}{ Sex } \\
\hline Male $(n=889,618)$ & $2423(0.27)$ & $638(0.07)$ \\
\hline Female $(n=1,896,884)$ & $6669(0.35)$ & $1072(0.06)$ \\
\hline Unknown $(n=137,817)$ & $363(0.26)$ & $60(0.04)$ \\
\hline \multicolumn{3}{|l|}{ Age, years } \\
\hline$<18(n=62,164)$ & $97(0.16)$ & $61(0.10)$ \\
\hline $18-44(n=516,973)$ & $1830(0.35)$ & $515(0.10)$ \\
\hline $45-64(n=838,942)$ & $2822(0.34)$ & $525(0.06)$ \\
\hline$\geq 65$ years $(n=481,932)$ & $868(0.18)$ & $98(0.02)$ \\
\hline Unknown $(n=1,024,308)$ & $3838(0.37)$ & $571(0.06)$ \\
\hline \multicolumn{3}{|l|}{ Region } \\
\hline Africa $(n=3438)$ & $7(0.20)$ & $1(0.03)$ \\
\hline The Americas $(n=2,433,422)$ & $8648(0.36)$ & $1523(0.06)$ \\
\hline Southeast Asia $(n=4372)$ & $2(0.05)$ & $3(0.07)$ \\
\hline Europe $(n=376,078)$ & $640(0.17)$ & $196(0.05)$ \\
\hline Eastern Mediterranean $(n=3252)$ & $5(0.15)$ & $1(0.03)$ \\
\hline Western Pacific $(n=103,757)$ & $153(0.15)$ & $46(0.04)$ \\
\hline \multicolumn{3}{|l|}{ Reporting years } \\
\hline $1995-1999(n=1367)$ & $0(0)$ & $0(0)$ \\
\hline $2000-2004(n=52,267)$ & $57(0.11)$ & $5(0.01)$ \\
\hline $2005-2009(n=284,580)$ & $659(0.23)$ & $183(0.06)$ \\
\hline 2010-2014 $(n=1,243,659)$ & $4928(0.40)$ & $782(0.06)$ \\
\hline 2015-2017 $(n=1,342,446)$ & $3811(0.28)$ & $800(0.06)$ \\
\hline \multicolumn{3}{|l|}{ Reporter } \\
\hline Healthcare professional $(n=1,413,828)$ & $3221(0.23)$ & $991(0.07)$ \\
\hline Consumer $(n=1,285,732)$ & $5600(0.44)$ & $633(0.05)$ \\
\hline Other $(n=29,983)$ & $110(0.37)$ & $32(0.11)$ \\
\hline Unknown $(n=194,776)$ & $524(0.27)$ & $114(0.06)$ \\
\hline
\end{tabular}

$A D R s$ adverse drug reactions was strongest for mAbs suppressing the immune system (ROR 1.9, 95\% CI 1.8-2.0) when compared with mAbs not directly targeting the immune system. The mAbs suppressing the immune system were further stratified according to their intended indication. The results show that the association with depression for mAbs used for the treatment of autoimmune diseases was stronger compared with mAbs not directly targeting the immune system (ROR 1.96, 95\% CI 1.84-2.10), whereas for mAbs used for the treatment of cancer, this difference was not seen (ROR 0.9, 95\% CI 0.8-1.0). The mAbs stimulating the immune system were all indicated for the treatment of cancer and hence were not further stratified.

For suicidal ideation and behavior the ROR (relative to bevacizumab) was highest for belimumab $(20.2,95 \% \mathrm{CI}$ 12.4-33.0) followed by natalizumab (12.0, 95\% CI 7.9-18.3) (Fig. 3). When grouping the mAbs based on their influence on the immune system, the ROR for suicidal ideation and behavior was highest for mAbs suppressing the immune system (3.6, 95\% CI 3.0-4.4) compared with mAbs not directly targeting the immune system (Fig. 4). When stratifying the mAbs suppressing the immune system by their intended indication, the ROR for mAbs used for the treatment of autoimmune diseases was higher compared with mAbs not directly targeting the immune system (3.8, 95 CI 3.1-4.7), whereas for mAbs used for the treatment of cancer, this difference was not seen (ROR 1.1, 95\% CI 0.7-1.5).

\section{Discussion}

The current study is, to our knowledge, the first to assess the potential risk of depression and suicidal ideation and behavior for the group of mAbs as a whole. The most relevant finding of this study is that mAb-induced depression and 
Fig. 1 Reporting odds ratios, relative to bevacizumab, of depression adverse drug reactions for the monoclonal antibodies separately. $R O R$ reporting odds ratio

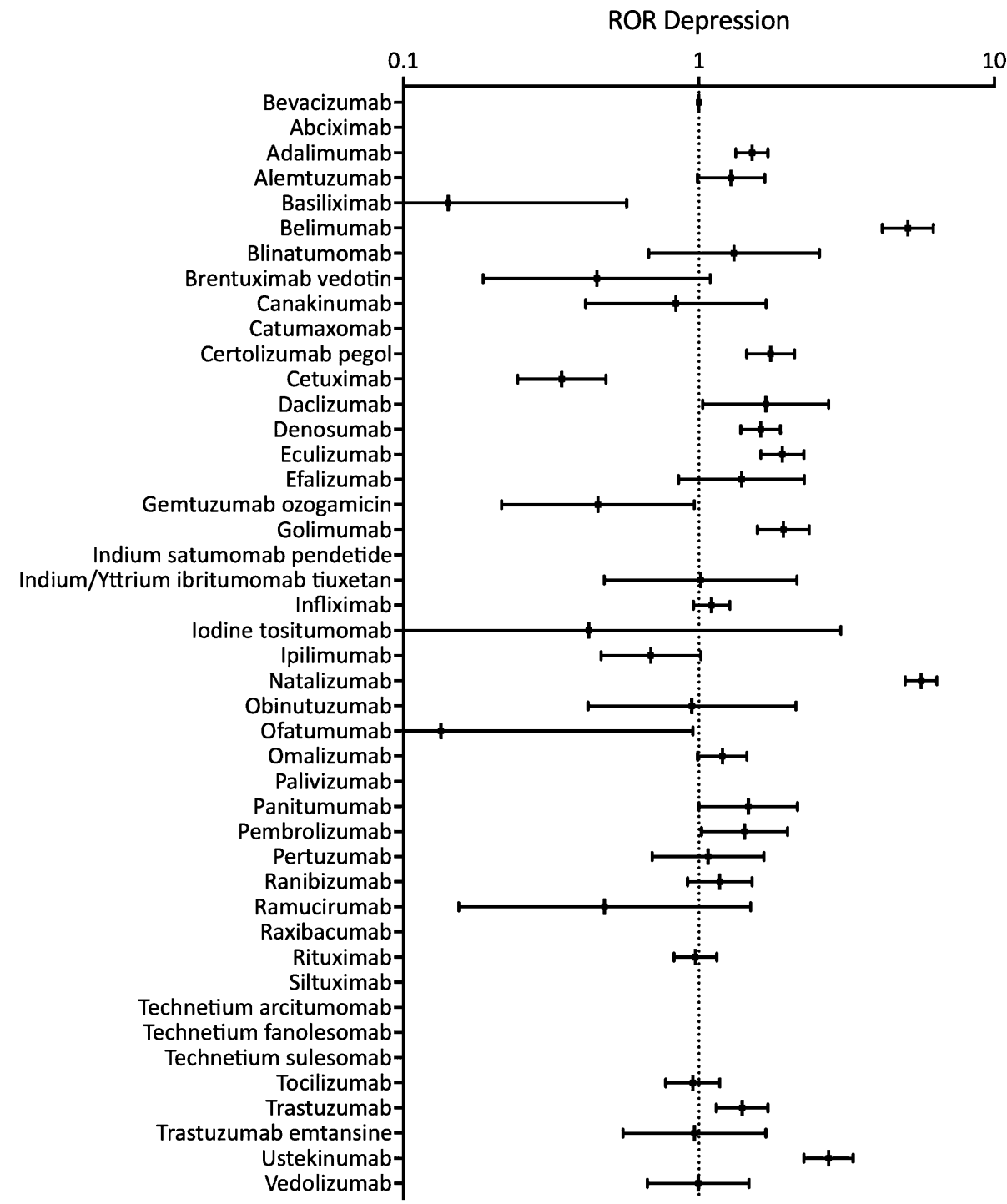

suicidal ideation and behavior seems to be associated with certain specific immune modulating properties.

Depression and suicidal ideation and behavior were most often reported for the mAbs belimumab and natalizumab. When the mAbs were grouped according to their influence on the immune system, we found that depression and suicidal ideation and behavior were more frequently reported for mAbs suppressing the immune system compared with mAbs that do not directly target the immune system. Further stratification by intended indication of the mAbs suppressing the immune system only showed this difference in mAbs used for the treatment of autoimmune diseases. When characterizing the reports, the most marked finding was that the proportion of consumer reports related to depression was approximately twofold higher compared with healthcare professionals.

The discrepancy in the reporting of depression by patients compared with healthcare professionals, as found in this study, is in line with previous studies showing that patients are more likely to report psychiatric ADRs compared with healthcare professionals [28, 29]. This may be explained by the nature of the events, as these are experienced by patients to have a direct impact on quality of life.

Belimumab and natalizumab showed the highest reporting of depression and suicidal ideation and behavior relative to bevacizumab. For belimumab, the potential risk of depression was seen in clinical trials and is listed in the product information [30, 31]. Furthermore, the pharmaceutical company is currently performing a study to further characterize this potential risk [30]. For natalizumab, studies have reported improvement in depression symptoms [32]. However, natalizumab has also been reported to cause suicidal ideation and behavior by inducing peripheral cell-mediated inflammation resulting in cytokine secretion (in particular, tumor necrosis factor [TNF]- $\alpha$ ) [10]. Our study shows a substantial number of reports for natalizumab, indicating that 
Fig. 2 Reporting odds ratios, relative to monoclonal antibodies not directly targeting the immune system, of depression adverse drug reactions for monoclonal antibodies, grouped by their influence on the immune system. ROR reporting odds ratio

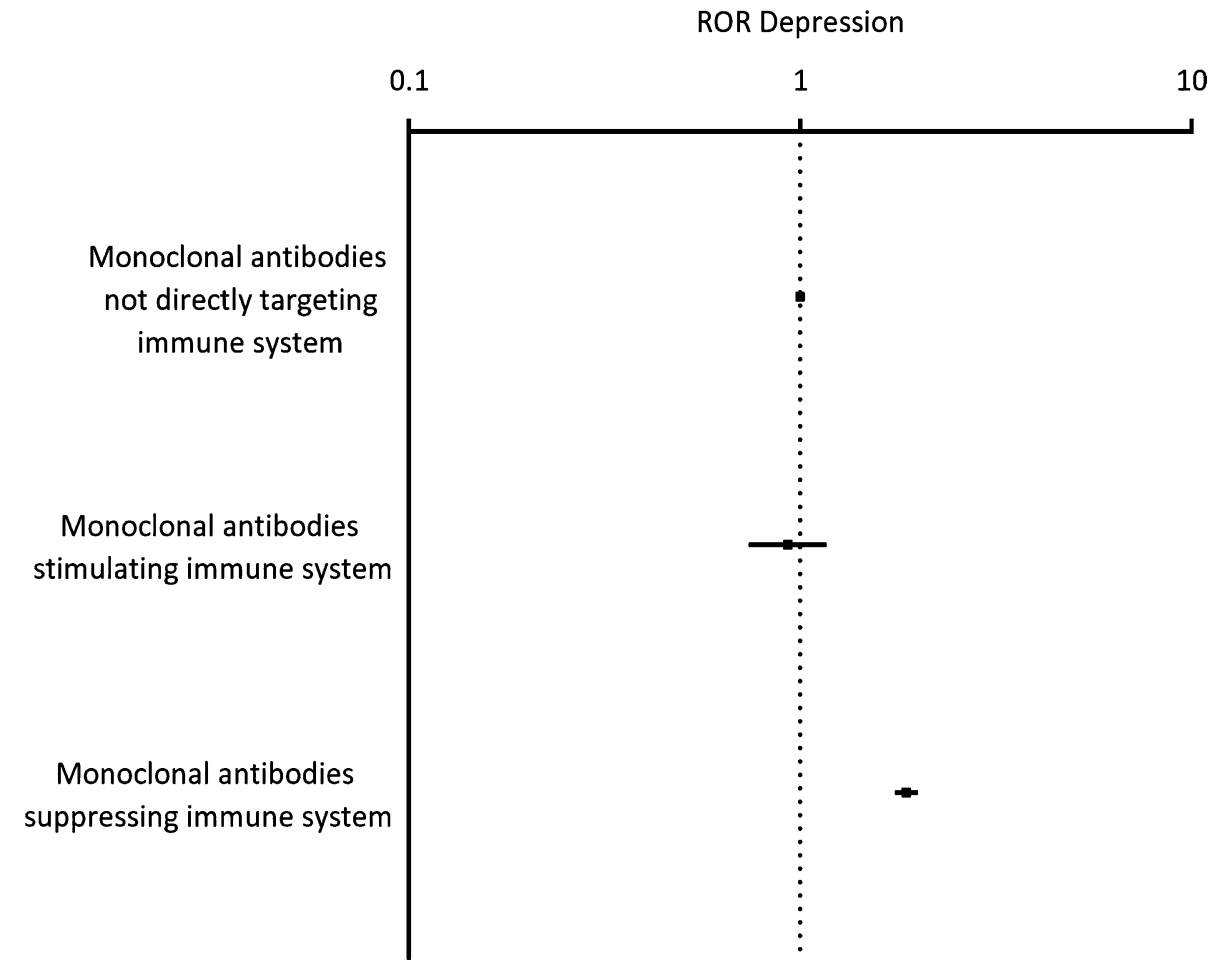

this potential risk should be taken into consideration when treating patients with natalizumab. Besides both agents suppressing the immune system, belimumab and natalizumab do not share further mechanistic commonalities. Due to the limited number of mAbs with more specific mechanistic commonalities (e.g. TNF $\alpha$ inhibitors, IL inhibitors), we were unable to clearly identify patterns in reporting for mAbs with these mechanistic commonalities. As a result, it is not possible to indicate if this potential risk should be monitored for in the postmarketing phase of new mAbs with a specific mode of action.

We also found that depression and suicidal ideation and behavior are more frequently reported for mAbs suppressing the immune system compared with mAbs that do not directly target the immune system. Different meta-analyses showed that inflammatory factors play a role in the pathophysiology of depression and suicidal ideation and behavior [15-20]. In general, it is assumed that patients with depression or suicidal ideation and behavior have increased levels of proinflammatory factors compared with healthy subjects. However, in meta-analyses, alterations in both pro-inflammatory and anti-inflammatory factors have been linked to these psychiatric disorders. In addition, the influence of psychiatric diseases has been reported for both low and high levels of pro-inflammatory factors [33]. The influence exerted by inflammatory factors involves a complicated process and full understanding of their role is lacking. This makes it challenging to fully explain the potential influence of mAbs on these inflammatory factors. In this study, we showed that depression and suicidal ideation and behavior are more frequently reported for mAbs suppressing the immune system compared with mAbs that do not directly target the immune system. This may be explained by the alterations in the levels of inflammatory factors caused by the mAbs.

When the analysis was stratified by the intended indication of the $\mathrm{mAb}$, the difference seen for mAbs suppressing the immune system was only seen in mAbs used for the treatment of autoimmune diseases and not those that suppress the immune system and are used for the treatment of cancer. The potential issue of confounding by indication should therefore be taken into consideration when interpreting these results. As previously described, the estimated prevalence of depression for both patients with cancer and autoimmune diseases exceeds the estimated prevalence in the general population; however, for the main indications of the mAbs suppressing the immune system included in our study, the prevalence of depression seems comparable. In the populations treated with mAbs used for the main autoimmune indications (rheumatoid arthritis, multiple sclerosis, Crohn's disease, psoriasis, and systemic lupus erythematosus), the prevalence of depression is similar and ranges from 20 to $30 \%$ [23, 34-37]. The main indications for the mAbs used for cancer were hematological malignancies, and the prevalence of depression is estimated to be approximately $20 \%$ in these patients [38].

The strength of this study is that the reports included where retrieved from VigiBase, the largest database containing ICSRs, and were therefore well-suited to obtain 
Fig. 3 Reporting odds ratios, relative to bevacizumab, of suicidal ideation and behavior adverse drug reactions for the monoclonal antibodies separately. $R O R$ reporting odds ratio

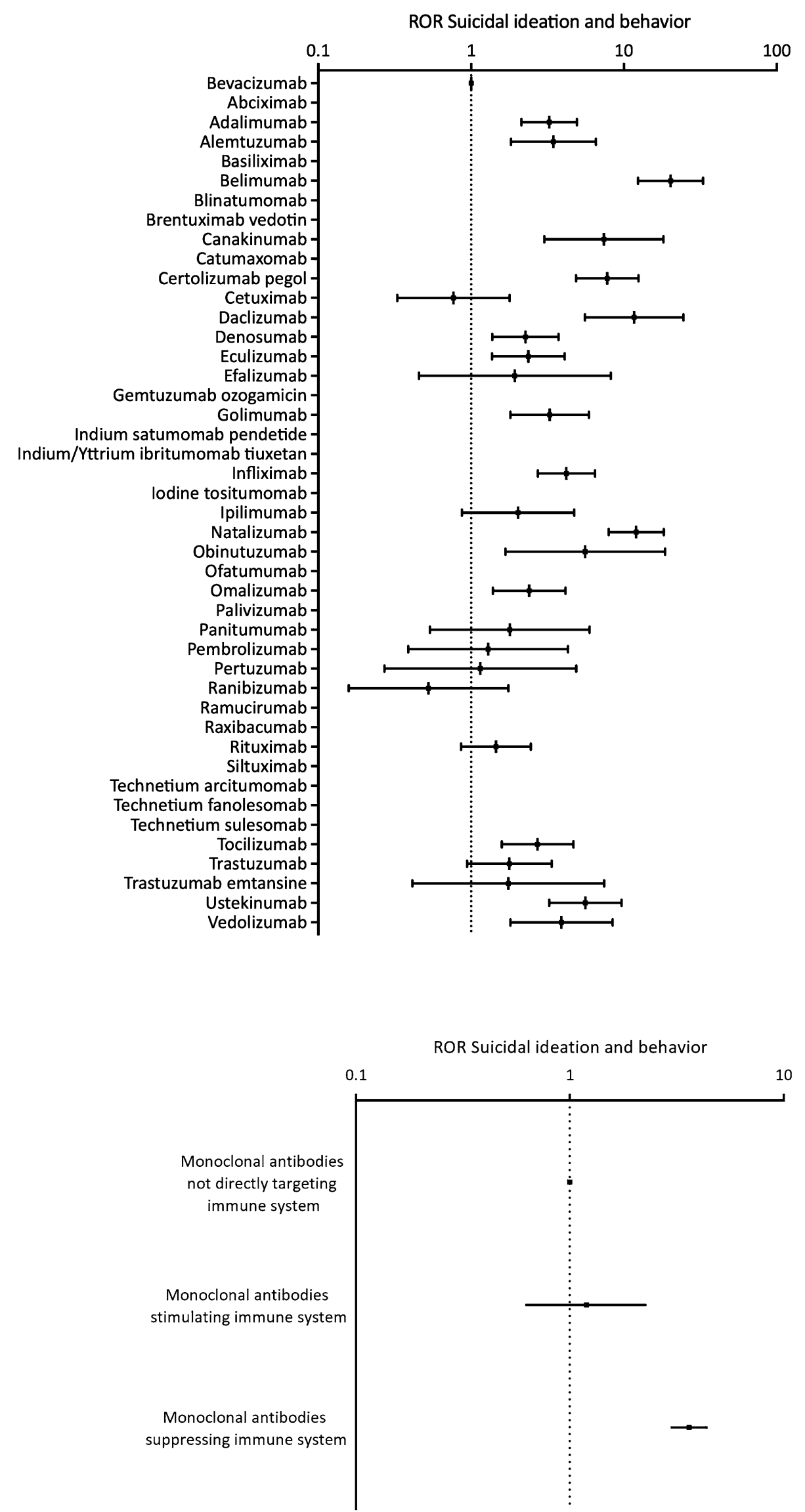

Fig. 4 Reporting odds ratios, relative to monoclonal antibodies not directly targeting the immune system, of suicidal ideation and behavior adverse drug reactions for monoclonal antibodies, grouped by their influence on the immune system. $R O R$ reporting odds ratio 
insight into reporting patterns, as well as for ADRs with a low reporting frequency, such as suicidal ideation and behavior. Furthermore, the analysis was not only performed for the mAbs separately but aimed to explore the reporting of depression and suicidal ideation and behavior to the mechanism of action of the mAbs.

\subsection{Limitations}

Several limitations are introduced when using data from a spontaneous reporting system. First, we did not have information on the number of patients exposed to the $\mathrm{mAbs}$ and the potential history of psychiatric disorders of the patients. In addition, we did not perform a formal causality assessment of the reports. Furthermore, the willingness to report depression and suicidal ideation and behavior may be different between the different disease categories. Therefore, the results of this study should be considered hypothesis-generating, and additional studies are needed to further characterize the potential risk of depression and suicidal ideation and behavior in patients treated with mAbs.

\section{Conclusion}

Events of depression and suicidal ideation and behavior are reported for mAbs, especially for mAbs that suppress the immune system and that are used for the treatment of autoimmune diseases. This further supports the link between inflammatory factors and the occurrence of depression and suicidal ideation and behavior. The present study provides important knowledge for future research in which, among others, the relation between the different inflammatory factors and the occurrence of depression and suicidal ideation and behavior, indications for use, and population characteristics should be studied to characterize and quantify this potential risk.

Acknowledgements The authors would like to thank the Uppsala Monitoring Centre for providing the data. The information in this paper does not represent the opinion of the World Health Organization, the Uppsala Monitoring Center, or national centers.

\section{Compliance with Ethical Standards}

Conflict of interest Lotte Minnema, Thijs Giezen, Patrick Souverein, Toine Egberts, Hubert Leufkens and Helga Gardarsdottir have no conflicts of interest that are directly relevant to the content of this study.

Funding No external sources of funding were used to assist in the preparation of this article.
OpenAccess This article is distributed under the terms of the Creative Commons Attribution-NonCommercial 4.0 International License (http://creativecommons.org/licenses/by-nc/4.0/), which permits any noncommercial use, distribution, and reproduction in any medium, provided you give appropriate credit to the original author(s) and the source, provide a link to the Creative Commons license, and indicate if changes were made.

\section{References}

1. Schmidt C. Suicidal thoughts end Amgen's blockbuster aspirations for psoriasis drug. Nat Biotechnol. 2015;33(9):894-5.

2. European Medicines Agency. European Public Assessment Report Kyntheum (brodalumab). 2017 [cited 26 Aug 2017]. Available at: http://www.ema.europa.eu/docs/en_GB/document_library/ EPAR_-_Public_assessment_report/human/003959/WC50023291 6.pdf.

3. Chen C, Itakura E, Nelson GM, Sheng M, Laurent P, Fenk LA, et al. IL-17 is a neuromodulator of Caenorhabditis elegans sensory responses. Nature. 2017;542(7639):43-8.

4. Silverstein NJ, Huh JR. Interleukin-17: why the worms squirm. Immunity. 2017;46(3):347-9.

5. Choi GB, Yim YS, Wong H, Kim S, Kim H, Kim SV, et al. The maternal interleukin-17a pathway in mice promotes autismlike phenotypes in offspring. Science. 2016;351(6276):933-9.

6. Wallace D, Navarra S, Petri M, Gallacher A, Thomas M, Furie $\mathrm{R}$, et al. Safety profile of belimumab: pooled data from placebocontrolled phase 2 and 3 studies in patients with systemic lupus erythematosus. Lupus. 2013;22(2):144-54.

7. Ellard R, Ahmed A, Shah R, Bewley A. Suicide and depression in a patient with psoriasis receiving adalimumab: the role of the dermatologist. Clin Exp Dermatol. 2014;39(5):624-7.

8. Eshuis EJ, Magnin KMMY, Stokkers PCF, Bemelman WA, Bartelsman J. Suicide attempt in ulcerative colitis patient after 4 months of infliximab therapy: a case report. J Crohns Colitis. 2010;4(5):591-3.

9. Mumoli L, Ciriaco M, Gambardella A, Bombardiere GN, Valentino $\mathrm{P}$, Palleria $\mathrm{C}$, et al. A possible case of natalizumab-dependent suicide attempt: a brief review about drugs and suicide. J Pharmacol Pharmacother. 2013;4(Suppl 1):S90-3.

10. Nagesh O, Bastiampillai T, Fisher L, Mohan T. Cyclical suicidal ideation following natalizumab infusion for multiple sclerosis. Aust N Z J Psychiatry. 2015;49(7):668-9.

11. Roblin X, Oltean P, Heluwaert F, Bonaz B. Panic attack with suicide: an exceptional adverse effect of infliximab. Dig Dis Sci. 2006;51(6): 1056

12. Bagley SC, Sirota M, Chen R, Butte AJ, Altman RB. Constraints on biological mechanism from disease comorbidity using electronic medical records and database of genetic variants. PLoS Comput Biol. 2016;12(4):e1004885.

13. Benros ME, Waltoft BL, Nordentoft M, Østergaard SD, Eaton WW, Krogh J, et al. Autoimmune diseases and severe infections as risk factors for mood disorders: a nationwide study. JAMA Psychiatry. 2013;70(8):812-20.

14. Dantzer R, O'Connor JC, Freund GG, Johnson RW, Kelley KW. From inflammation to sickness and depression: when the immune system subjugates the brain. Nat Rev Neurosci. 2008;9(1):46-56.

15. Köhler CA, Freitas TH, Maes M, de Andrade NQ, Liu CS, Fernandes BS, et al. Peripheral cytokine and chemokine alterations in depression: a meta-analysis of 82 studies. Acta Psychiatr Scand. 2017;135(5):373-87. 
16. Liu Y, Ho RC-M, Mak A. Interleukin (IL)-6, tumour necrosis factor alpha (TNF- $\alpha$ ) and soluble interleukin-2 receptors (sIL-2R) are elevated in patients with major depressive disorder: a metaanalysis and meta-regression. J Affect Disord. 2012;139(3):230-9.

17. Eyre HA, Air T, Pradhan A, Johnston J, Lavretsky H, Stuart MJ, et al. A meta-analysis of chemokines in major depression. Prog Neuropsychopharmacol Biol Psychiatry. 2016;68:1-8.

18. Dowlati Y, Herrmann N, Swardfager W, Liu H, Sham L, Reim EK, et al. A meta-analysis of cytokines in major depression. Biol Psychiatry. 2010;67(5):446-57.

19. Hiles SA, Baker AL, de Malmanche T, Attia J. A meta-analysis of differences in IL- 6 and IL-10 between people with and without depression: Exploring the causes of heterogeneity. Brain Behav Immun. 2012;26(7):1180-8.

20. Haapakoski R, Mathieu J, Ebmeier KP, Alenius H, Kivimäki M. Cumulative meta-analysis of interleukins 6 and $1 \beta$, tumour necrosis factor $\alpha$ and C-reactive protein in patients with major depressive disorder. Brain Behav Immun. 2015;49:206-15.

21. European Medicines Agency. Product Information Herceptin (trastuzumab). 2017. Available at: http://www.ema.europa.eu/ docs/en_GB/document_library/EPAR_-_Product_Information/ human/000278/WC500074922.pdf.

22. Pitman A, Suleman S, Hyde N, Hodgkiss A. Depression and anxiety in patients with cancer. BMJ. 2018;361:k1415.

23. Matcham F, Rayner L, Steer S, Hotopf M. The prevalence of depression in rheumatoid arthritis: a systematic review and metaanalysis. Rheumatology. 2013;52(12):2136-48.

24. Lindquist M. VigiBase, the WHO global ICSR database system: basic facts. Drug Inf J. 2008;42(5):409-19.

25. MedDRA Maintenance and Support Services Organization. MedDRA, Medical Dictionary for Regulatory Activities [cited 29 Aug 2017]. Available at: https://www.meddra.org/.

26. Montastruc J-L, Sommet A, Bagheri H, Lapeyre-Mestre M. Benefits and strengths of the disproportionality analysis for identification of adverse drug reactions in a pharmacovigilance database. Br J Clin Pharmacol. 2011;72(6):905-8.

27. van Puijenbroek EP, Bate A, Leufkens HGM, Lindquist M, Orre R, Egberts ACG. A comparison of measures of disproportionality for signal detection in spontaneous reporting systems for adverse drug reactions. Pharmacoepidemiol Drug Saf. 2002;11(1):3-10.
28. Banovac M, Candore G, Slattery J, Houÿez F, Haerry D, Genov $\mathrm{G}$, et al. Patient reporting in the EU: analysis of EudraVigilance data. Drug Saf. 2017;40(7):629-45.

29. Avery AJ, Anderson C, Bond CM, Fortnum H, Gifford A, Hannaford PC, et al. Evaluation of patient reporting of adverse drug reactions to the UK 'Yellow Card Scheme': literature review, descriptive and qualitative analyses, and questionnaire surveys. Health Technol Assess Winch Engl. 2011;15(20):1-234, iii-iv.

30. European Medicines Agency. European Public Assessment Report Benlysta (belimumab). 2011 [cited 26 Mar 2018]. Available at: http://www.ema.europa.eu/docs/en_GB/document_libra ry/EPAR_-_Public_assessment_report/human/002015/WC500 110152.pdf.

31. European Medicines Agency. Product Information Benlysta (belimumab). 2017. Available from: http://www.ema.europa.eu/docs/ en_GB/document_library/EPAR_-_Product_Information/human /002015/WC500110150.pdf.

32. Hoepner R, Faissner S, Salmen A, Gold R, Chan A. Efficacy and side effects of natalizumab therapy in patients with multiple sclerosis. J Cent Nerv Syst Dis. 2014;6:41-9.

33. Tonelli LH. Neuroinflammation in suicide: too little may be just as bad as too much. Acta Psychiatr Scand. 2015;131(4):242-3.

34. Dowlatshahi EA, Wakkee M, Arends LR, Nijsten T. The prevalence and odds of depressive symptoms and clinical depression in psoriasis patients: a systematic review and meta-analysis. J Invest Dermatol. 2014;134(6):1542-51.

35. Zhang L, Fu T, Yin R, Zhang Q, Shen B. Prevalence of depression and anxiety in systemic lupus erythematosus: a systematic review and meta-analysis. BMC Psychiatry. 2017;17(1):70.

36. Neuendorf R, Harding A, Stello N, Hanes D, Wahbeh H. Depression and anxiety in patients with Inflammatory Bowel Disease: a systematic review. J Psychosom Res. 2016;87:70-80.

37. Marrie RA, Reingold S, Cohen J, Stuve O, Trojano M, Sorensen $\mathrm{PS}$, et al. The incidence and prevalence of psychiatric disorders in multiple sclerosis: a systematic review. Mult Scler. 2015;21(3):305-17.

38. Linden W, Vodermaier A, MacKenzie R, Greig D. Anxiety and depression after cancer diagnosis: Prevalence rates by cancer type, gender, and age. J Affect Disord. 2012;141(2):343-51. 